\title{
Local norms of cheating and the cultural evolution of crime and punishment: A study of two urban neighborhoods
}

The prevalence of antisocial behavior varies across time and place. The likelihood of committing such behavior is affected by, and also affects, the local social environment. To further our understanding of this dynamic process, we conducted two studies of antisocial behavior, punishment, and social norms. These studies took place in two neighborhoods in Newcastle Upon Tyne, England. According to a previous study, Neighborhood A enjoys relatively low frequencies of antisocial behavior and crime and high levels of social capital. In contrast, Neighborhood B is characterized by relatively high frequencies of antisocial behavior and crime and low levels of social capital. In Study 1, we used an economic game to assess neighborhood differences in theft, third-party punishment (3PP) of theft, and expectation of 3PP. Participants also reported their perceived neighborhood frequency of cooperative norm violation ("cheating"). Participants in Neighborhood B thought that their neighbors commonly cheat but did not condone cheating. They stole more money from their neighbors in the game, and were less punitive of those who did, than the residents of Neighborhood A. Perceived cheating was positively associated with theft, negatively associated with the expectation of 3PP, and central to the neighborhood difference. Lower trust in one's neighbors and a greater subjective value of the monetary cost of punishment contributed to the reduced punishment observed in Neighborhood B. In Study 2, we examined the causality of cooperative norm violation on expectation of 3PP with a norms manipulation. Residents in Neighborhood B who were informed that cheating is locally uncommon were more expectant of 3PP. In sum, our results provide support for three potentially simultaneous positive feedback mechanisms by which the perception that others are behaving antisocially can lead to further antisocial behavior: 1) motivation to avoid being suckered, 2) decreased punishment of antisocial behavior, and 3) decreased expectation of punishment of antisocial behavior. Consideration of these mechanisms and of norm psychology will help us to understand how neighborhoods can descend into an antisocial 
culture and get stuck there.

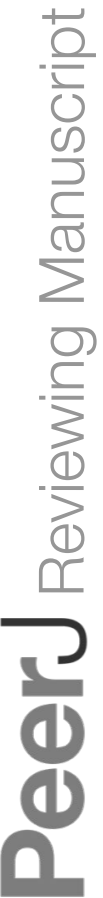

PeerJ reviewing PDF | (v2014:03:1718:1:1:NEW 2 Jun 2014) 
1 Authors: Kari Britt Schroeder ${ }^{1,2}$, Gillian V. Pepper $1^{1}$, Daniel Nettle ${ }^{1}$

2 Corresponding author: Kari Britt Schroeder, Department of Psychological and Brain Sciences,

3 Boston University, 64 Cummington Mall, Boston, MA, 02215 USA,

4 kari.britt.schroeder@gmail.com

5

6

7

$18{ }^{1}$ Centre for Behaviour and Evolution, Newcastle University, Newcastle Upon Tyne, United

19 Kingdom

$20{ }^{2}$ Department of Psychological and Brain Sciences, Boston University, Boston, MA, United States 21 of America 


\section{Introduction.}

23 Why do humans behave antisocially? The converse of this question - why humans behave

24 prosocially - has been studied extensively by experimental economists, and determinants of

25 prosocial behavior may be mirror images of determinants of antisocial behavior. One proximate

26 explanation for prosocial behavior is punishment; i.e., people will behave prosocially if not doing

27 so results in punishment. Empirical evidence for this comes from economic games. Using a

28 repeated public goods game, Yamagishi (1986) and Fehr and Gächter (2000) showed that the

29 opportunity for players to fine each other on the basis of contribution behavior can stabilize

30 contributions to the public good at a high level. Following this, the cross-cultural covariation of

31 prosocial behavior and punishment has received substantial interest (Henrich et al. 2006;

32 Herrmann, Thöni \& Gächter 2008). Considerable local variation in prosociality has also been

33 observed (Wilson, O'Brien \& Sesma 2009; Nettle, Colléony \& Cockerill 2011; Lamba \& Mace

34 2011), yet the question of whether prosocial behavior and punishment positively covary at the

35 local level has spurred little research among experimental economists (but see Kocher,

36 Martinsson \& Visser (2012)).

37 However, the related question of whether antisocial behavior and a lack of punishment

38 positively covary at the neighborhood level has generated substantial research within the field of

39 sociology. Social disorganization theory posits that poverty, residential mobility, and family

40 disruption can diminish the capacity a community has for creating relationships and establishing

41 shared social norms. This low level of 'social capital' can lead to increased crime and delinquency

42 via reduced collective action ( Shaw \& McKay 1942; Sampson \& Groves 1989; Bursik \&

43 Grasmick 1993; Sampson, Raudenbush \& Earls 1997). Without trust and shared behavioral

44 expectations, residents have decreased capacity to enforce desirable behavior through informal

45 social control (i.e., informal surveillance and/or intervention by residents) (Sampson, Morenoff \&

46 Gannon-Rowley 2002). 
Of interest to researchers in both of these fields is how the local social environment can

48 evolve over time to become more prosocial or more antisocial. This requires an understanding of

49 the dynamic relationship between individual decisions (as typically studied by experimental

50 economists) and the local social environment (as typically studied by sociologists). That is,

51 individual decisions can be influenced by empirical expectations of the behavior of others in the

52 local social environment (Bichierri and Xiao 2009). These decisions, as manifest in observable

53 behavior, then become part of the local social environment. Others will form expectations on the

54 basis of their perception of the local environment and possibly alter their own behavior. That such

55 a dynamic relationship exists is suggested by, for example, the interdependence of individual

56 decisions to commit crimes (Glaeser, Sacerdote \& Scheinkman 1996).

57 In this paper, we attempt to bridge these two approaches of experimental economics and

58 sociology and increase our understanding of the dynamic relationship between individual

59 decisions and the social environment. We do so through consideration of the role of the

60 individual's expectation of others' cooperative behavior - that is, the role of perceived local

61 norms of cooperative behavior. Cialdini, Reno \& Kallgren distinguish between injunctive norms

62 and descriptive norms (1990). Injunctive norms convey how people should behave. Descriptive

63 norms, on the other hand, illustrate how most people actually do behave.

64 It is readily apparent that cooperative descriptive norms should be informative as to

65 people's expectation of cooperation. However, cooperative descriptive norms may also be

66 informative as to people's expectation of punishment for cooperative norm violation or antisocial

67 behavior, particularly when there is a mismatch between injunctive and descriptive norms. A lack

68 of alignment between injunctive and descriptive cooperative norms is implicit in broken windows

69 theory - the idea that signs of social and physical disorder invite criminal behavior - in part

70 because disorder is a cue that social control is lax (Kelling \& Wilson 1982). This

71 mechanism for the 'spread of disorder' was elegantly tested by Keizer, Lindenberg \& Steg (2008), 
72 who created public spaces in which an explicit injunctive norm was violated - e.g., a littered

73 space (conveying a descriptive norm) next to a sign telling people not to litter (injunctive norm) -

74 thereby communicating a lack of adherence to the injunctive norm and experimentally inducing

75 further antisocial behavior. These results suggest that signs that others are flouting injunctive

76 cooperative norms may serve as cues that antisocial behavior will not be punished. However, this

77 remains a largely untested explanation of these results and of the broken windows effect in

78 general (Traxler and Winter 2012; but see Lochner (2007)).

Important to the studies we present in this paper, the work of Keizer, Lindenberg \& Steg

$80(2008 ; 2013)$ also demonstrated the possibility for 'cross-norm effects' - that is, the focus of the

81 injunctive and descriptive norms was different from the behavioral outcome assessed by the

82 researchers. Some of the observed cross-norm effects included public versus private goods. For

83 example, graffiti and litter (destruction of a public good) each resulted in an increase in theft of

84 an envelope with money in it (Keizer, Lindenberg \& Steg 2008). In another set of experiments,

85 these same authors also demonstrated cross-norm effects for the restoration of a public good and

86 prosocial behavior targeted at an individual; garbage bags on the street - in violation of city

87 ordinance - resulted in a decrease in posting of a letter dropped next to a postbox (Keizer,

88 Lindenberg \& Steg 2013).

89 Thus, studying injunctive and descriptive cooperative norms presents a way to assess

90 individual perceptions of environmental variation in cooperative and, potentially, punitive

91 behavior. It also offers a way to study how the social environment affects the behavior of the

92 individual and individual's behavior in turn affects the social environment, by conveying

93 information about descriptive norms. It is particularly appropriate when the focus is on local

94 (rather than large-scale) variation in prosocial or antisocial behavior, as injunctive norms may be

95 more similar in areas where people share common culture and history, while descriptive norms

96 may still vary. Given a general consensus on injunctive norms, the emphasis can then be on 
perceived deviation from the injunctive norms.

98

99 The Studies

100 Our studies were set in two nearby neighborhoods in Newcastle Upon Tyne, England, that

101 we expected to have similar injunctive cooperative norms based on a shared cultural history.

102 These two neighborhoods are similar in size, physical layout, and ethnic composition yet differ

103 dramatically in rates of antisocial behavior and socioeconomic deprivation. While Neighborhood

104 A is relatively affluent, Neighborhood B has experienced high rates of unemployment, physical

105 decay, massive depopulation, and crime, following the collapse of mining and shipbuilding

106 industries (see Nettle, Colléony \& Cockerill (2011) and citations therein). In an earlier study,

107 Nettle, Colléony \& Cockerill used surveys, a Dictator Game, behavioral observation, and field

108 experiments to reveal substantially less antisocial behavior, more social capital, and more

109 prosocial behavior in Neighborhood A than B (2011).

110 Here, we return to these neighborhoods to investigate whether individual decisions to

111 engage in antisocial behavior and norm enforcement vary by neighborhood. To do so, we

112 evaluated antisocial behavior, punishment, and expectation of punishment in an economic game.

113 We used a questionnaire to investigated whether neighborhood differences in antisocial behavior,

114 punishment, and expectation of punishment could be explained by neighborhood differences in

115 trust and local descriptive cooperative norms. Study 1 was observational and aimed to document

116 and explain differences in perceptions and behaviors between the neighborhoods. Study 2

117 introduced a novel experimental methodology to manipulate perceived injunctive norm

118 adherence, allowing us to make causal inferences. We assessed whether information on injunctive

119 cooperative norm adherence altered expectations of punishment for antisocial behavior.

\section{Study 1.}


122 Camerer and Fehr suggest that a real-world example of a third-party punishment game (3PP

123 game) (Fehr \& Fischbacher 2004) is scolding of a neighbor for treating another person

124 unacceptably (2004). In this study, we administered a 3PP game along with a questionnaire (see

125 Supplemental Information, SI). Our variant of the game, which was played among residents

126 within each neighborhood, enabled us to study differences between the neighborhoods in

127 antisocial behavior and punishment for antisocial behavior. Player 1 was given the opportunity to

128 steal from Player 2. Player 3 was given the opportunity to fine Player 1 if she took money from

129 Player 2. Player 2 indicated whether she thought Player 3 would fine Player 1 if Player 1 took

130 half of Player 2's money.

131 We used Player 1 and Player 2 decisions to assess whether residents of Neighborhood B

132 were 1) more likely to behave antisocially and 2) less likely to expect someone in their

133 neighborhood to intervene in antisocial behavior. In conjunction with the questionnaire, we also

134 used Player 1 and 2 decisions to investigate 3) whether perceived local cooperative norm

135 violation could explain the the hypothesized neighborhood differences in individual antisocial

136 decisions and 4) punitive expectations.

137 We used Player 3 game decisions and the questionnaire to assess 1) whether residents of

138 Neighborhood A were more willing than those of B to punish antisocial behavior in their

139 neighborhood, and 2) whether, following social disorganization theory, neighborhood trust can

140 explain the hypothesized relationship between neighborhood and punitive behavior.

141

142 Study 1 Methods.

143 Sampling. The Ethics Committee of the Newcastle University Faculty of Medical Sciences

144 approved the study protocol (Protocol \#00503/2011 and Amendment \#00503_1/2012). Written

145 consent was not obtained because it would have been the only record of participation. We

146 conducted the study from July 2012 to December 2012. A maximum of one participant per 
147 household was drawn from the electoral roll. Potential participants received a hand-delivered

148 envelope with a cover letter describing the study, packet (questionnaire, explanation of the game,

149 and game), and stamped return envelope. A minority of envelopes were delivered by subjects in

150 another study (Nettle et al. 2014). We avoided sampling adjacent households and households

151 sampled by Nettle et al. (Nettle, Colléony \& Cockerill 2011).

152

153 Questionnaire. From the questionnaire, we recorded each participant's age and sex.

154 Trust. We asked individuals how much they trust people in their neighborhood, on a 10-

155 point scale $(10=$ most trusting $)$.

156 Civic norms: condoned and perceived cheating. We asked individuals about both

157 injunctive and descriptive civic norms (SI). For the injunctive norms, we described three

158 behaviors and asked whether it is Never OK to do this behavior, Always OK, or somewhere in

159 between. Answers were constrained to a 10-point scale ( $1=$ 'Always OK' and $10=$ 'Never OK').

160 The behaviors were 1) cheating the benefits system, 2) avoiding a fare on public transport, and 3)

161 cheating on taxes. Condoned cheating is the average across behaviors. Larger values indicate

162 that cheating on public goods is condoned. Note that condoned cheating is similar to the 'norms

163 of civic cooperation' (Knack \& Keefer 1997; Herrmann, Thöni \& Gächter 2008) derived from the

164 World Values Survey.

165 For the descriptive norm, we asked individuals whether they think many people in their

166 neighborhood would do this behavior $(1=$ 'No one would' and $10=$ 'Everyone would'). We

167 averaged across these responses to arrive at perceived cheating. Larger values indicate that

168 neighborhood cheating on public goods is perceived as more common.

169 We note that the cooperative norms used in the questionnaire pertain to public goods,

170 while the possibility for antisocial behavior in the game is directed at a single person. However, 
171 as mentioned in the introduction, previous studies have experimentally demonstrated 'cross-norm

172 effects' wherein violation or restoration of a public good induced antisocial or prosocial behavior, 173 respectively, directed at a single individual (Keizer, Lindenberg \& Steg 2008; Keizer, Lindenberg 174 \& Steg 2013).

176 The 3PP game. Participants read instructions for the game, which followed the questionnaire, and 177 then worked through examples (see SI). (From this, we had responses to six test questions.) They 178 were told that after receiving the packet in the post, we would determine the game outcome and 179 then deliver their cash payoff along with a $£ 5$ payment for completing the survey.

180 The game worked as follows: All three players received an initial allocation of $£ 10$, to be 181 paid after the decisions of all three players had been submitted. Player 1 had to decide how many 182 pounds (integer from 0 to 10) to take from Player 2. If Player 1 took money from Player 2, Player 1833 had to decide whether to fine Player 1. We used the strategy method for Player 3. Player 3 had

184 to decide, for each amount greater than 0 that Player 1 could take, whether to pay to fine Player 1. 185 Therefore, Player 3 had to make 10 choices, each corresponding to an amount that Player 1 might 186 take from Player 2. The cost of the fine to Players 1 and 3 was constant (Player 3 paid $£ 2$ to make 187 Player 1 lose £6). Player 2 could not make a choice in the game. We asked Player 2 to indicate 188 whether she thought Player 3 would fine Player 1 if she took $£ 5$ from her (SI).

189 Game behaviors are thus: theft (an integer from 0 to 10 representing the amount of money 190 Player 1 took from Player 2), expect 3PP (whether Player 2 expected Player 3 to punish Player 1

191 if she took $£ 5$ ), and punitiveness (an integer from 0 to 10; this is the total number of potential

192 thefts, from one pound $£ 1$ to $£ 10$, that Player 3 would punish).

193

194 Subjective value of money. We expected the subjective value of money to differ between 
195 neighborhoods and impact game behavior. Therefore, following the game, we asked how much of

196 a difference, on a scale of one to 10, an amount of money $x$ would make to their weekly budget,

197 where $x$ was $£ 1$ for Player 1 (value $£ 1$ ) and $£ 2$ for Players 2 and 3 (value $£ 2$ ). After

198 commencement of data collection, we revised the packets for Player 1 to include $x=£ 10$. Thus,

199 for some Player 1s we also have value $£ 10$.

200

201 Statistical analyses. The majority of responses can be considered discrete ordered choices. Thus,

202 to assess neighborhood differences in game behavior, trust, cooperative norms, and the value of

203 money, we analyzed the data with ordered logistic regression. The exception to this is game

204 behavior for Player 2, for which we used binary logistic regression. We compared the fit of

205 different models with the Akaike information criterion (AIC) (Akaike 1974). Ordered and

206 binomial logistic regression analyses and plotted predictions (i.e., the predicted value based on

207 the fitted model and the data used to fit the model) were produced in the R statistical and

208 computing environment (R Core Team 2012) with the following packages: MASS (Venables \&

209 Ripley 2002), rethinking (McElreath 2012), beeswarm (Ecklund 2012), and ggplot2 (Wickham

210 2009). Note that plotted predictions for theft and punitiveness are both $(0,8)$. For each of these

211 game behaviors, two possible values were not observed (3 and 8 for theft, 2 and 9 for

212 punitiveness); thus, for prediction we condensed the ranges. We report Odds Ratios (ORs) for a

213 unit increase in the outcome for each unit increase of the predictor variable, accompanied by $95 \%$

214 confidence intervals.

215

216 Study 1 Results.

217 Participants. We achieved sample sizes of 40 (16 male), 44 (22 male), and 49 (23 male) for

218 Players 1, 2, and 3, respectively, in Neighborhood A and 34 (12 male), 43 (23 male), and 50 (23 
219 male) in B (Table S1). Every week, new players from each neighborhood were combined into

220 triads, and we determined game outcome from their decisions. For incomplete triads, players

221 were drawn at random from all previous neighborhood players. We delivered to participants: the

222 game outcome, debriefing sheet, money received from the game, and $£ 5$ for participating. The

223 mean payoff from the game is £9.26 $(\sigma=£ 3.49)$ in Neighborhood A and £9.16 in B $(\sigma=£ 4.13)$.

224 Descriptive statistics and neighborhood comparisons for key variables are in Table 1. We also

225 report descriptive statistics in the text to assist the reader.

226

227 Trust. Participants in Neighborhood A indicated far higher trust neighbors (median 8 on a scale of

228 1:10, median absolute deviation (MAD) 1) than did participants in B (median 5, MAD 2) (Table

229 1) (OR 18.8, 95\% CI 10.8-32.8).

230

231 Punishment of antisocial behavior. As predicted, participants in Neighborhood A were more

232 punitive than those in B (Fig. 1) (OR 3.3, 95\% CI 1.6-7.0). Median punitiveness is 6 (MAD 4)

233 and 3 (MAD 3) for Neighborhoods A and B, respectively. Thus, more participants in

234 Neighborhood A indicated that they would pay $£ 2$ to fine Player 1 for a greater number of

235 potential thefts.

236 The subjective cost of punishment in the game, value $£ 2$, had a negative effect upon

237 punitiveness (OR 0.7, 95\% CI 0.6-0.9) and was larger for participants in Neighborhood B than A

238 (Table 1). However, participants in Neighborhood A were still more punitive than those in B

239 when we include value $£ 2$ in the model (OR 2.1, 95\% CI 0.9-4.6). This result is robust to the

240 inclusion of additional covariates age, male, and test questions (OR 2.9, 95\% CI 1.2-7.2).

241 Based on social disorganization theory, we hypothesized that greater trust among residents

242 of Neighborhood A would partially explain the increased willingness of residents to engage in

$2433 \mathrm{PP}$ of antisocial behavior. Individuals who reported greater trust neighbors were slightly more 
244 punitive (OR 1.15, 95\% CI 0.99-1.32). The relationship between trust and punitiveness is less

245 robust to the inclusion of value $£ 2$ (OR 1.09, 95\% CI 0.94-1.27); however, including an

246 interaction between value $£ 2$ and trust neighbors improves model fit (AIC of 380.13 compared to

$247384.49)$.

248 Predictions from this model including the interaction are shown in Fig. 2. value $£ 2$ still

249 has a negative effect on punitiveness, but the slope is steeper for participants with high trust

250 neighbors. Thus, participants with high trust neighbors are more punitive than those with low

251 trust neighbors when value $£ 2$ is small, but less punitive when it is large. Neighborhood is no

252 longer a reliable predictor of punitiveness when the interaction is included in the model (OR 1.8,

$25395 \%$ CI 0.7, 5.7), nor does model fit improve with the addition of neighborhood (AIC $=380.67$ ).

255 Civic norms: condoned and perceived cheating. In both neighborhoods, most participants

256 indicated that it is not acceptable to cheat on public goods. We observed little variation in

257 injunctive norms across cooperative behaviors (Table 1). Nor did we detect a clear difference

258 between neighborhoods with respect to specific injunctive norms (Fig. 3, Table 1) or condoned

259 cheating (i.e., the within-participant mean of injunctive norms).

260 However, there was a dramatic difference between neighborhoods with respect to

261 perceived cheating. Participants in Neighborhood B indicated that more of their neighbors would

262 cheat on a public good than those in A (median 3.00, MAD 0.67 for A; median 5.50, MAD 1.83

263 for A) (Fig. 3, Table 1). Participants who thought more of their neighbors cheat on public goods

264 were also less trusting of their neighbors (OR 0.54, 95\% CI 0.48-0.62).

265 Juxtaposition of condoned cheating and perceived cheating reveals that although

266 participants in Neighborhood B tended to state that many of their neighbors cheat on public

267 goods, we lack strong evidence that they view this behavior as more acceptable than those in A.

268 This fits with our prior expectation that injunctive cooperative norms would be similar in 
269 Neighborhoods A and B. We therefore use perceived cheating as a within-participant measure of

270 perceived local cooperative norm violation, or deviation from the injunctive cooperative norm.

271

272 Antisocial behavior. Participants in Neighborhood B took more from their neighbors in the game.

273 Theft is also more variable in Neighborhood B than A. The median value of theft is 5 in

274 Neighborhood B (MAD 5), compared to 0 in A (MAD 0) (odds that theft is greater in

275 Neighborhood B: OR 2.9, 95\% CI 1.2-7.1). The neighborhood difference in theft is robust to the

276 inclusion of age, male, and value $£ 1$ (OR 2.8, 95\% 2.5-6.9). For the reduced dataset for which we

277 had data on value $£ 10$ (40 participants, 23 from Neighborhood A), substituting this variable in the

278 model increases the odds that a participant in B stole more in the game (OR 4.1, 95\% CI 0.9-

279 17.5). Inclusion of test questions in the model reduces confidence in the neighborhood difference

280 in theft (OR 2.1, 95\% 0.8-5.8). However, incomplete test questions are heavily patterned for

281 Player 1; only participants in Neighborhood B for whom theft $>0$ did not complete the

282 questions. Irrespective of the participant's comprehension of the entire game, the opportunity for

283 Player 1 to behave antisocially (the outcome of interest to us) should be very clear from the

284 packet (i.e., "How many pounds do you choose to take from Player B? ") (SI).

285 As expected, perceived cheating is a robust predictor of theft, even controlling for value

$286 £ 1$ (Fig. 4; OR 1.3, 95\% CI 1.0-1.6). When both neighborhood and perceived cheating are

287 considered in the same model, neither is a reliable predictor of theft. Nor does AIC offer strong

288 support for a single model (235.40 for the model with perceived cheating, 234.67 for

289 neighborhood, and 234.60 for perceived cheating + neighborhood). This suggests that to

290 understand the greater theft in Neighborhood B, we need to consider perceived cheating.

291

292 Expectation of $3 P P$. We asked Player 2 whether she thought Player 3 would fine Player 1 if

293 Player 1 took $£ 5$ from her (expect 3PP). Contrary to our expectations, neighborhood was not a 
294 reliable predictor of expect 3PP. Of participants in Neighborhood A, 36.36\% expected 3PP,

295 compared to $30.23 \%$ of participants from Neighborhood B (OR 1.2, 95\% CI 0.5-3.2). However,

296 as predicted, we did observe a negative relationship between perceived cheating and expect $3 P P$

297 (Fig. 5; OR 0.8, 95\% CI 0.6-1). This relationship does not change with inclusion of value $£ 2$ as a

298 proxy for the local subjective value of $£ 2$ (OR $0.8,95 \%$ CI $0.6-1$ ).

300 Study 1 Summary and Discussion.

301 Study 1 reveals that individual perceptions of local cooperative descriptive norms (i.e., perceived

302 cheating) vary dramatically by neighborhood, in concordance with previous observations of

303 neighborhood discrepancies in antisocial behavior (including crime), prosocial behavior, and

304 social capital (Nettle, Colléony \& Cockerill 2011). Participants in Neighborhood B were far more

305 likely than those in A to think that more of their neighbors behave uncooperatively. We could not,

306 however, attribute this to a neighborhood difference in injunctive cooperative norms. Thus, a

307 perceived lack of adherence to injunctive cooperative norms was pervasive in Neighborhood B.

308 This general perception in Neighborhood B that others are behaving antisocially appears

309 justifiable: participants in Neighborhood B stole more money in the game. However, the results

310 of our analyses suggest that this neighborhood difference in theft in the game can be explained by

311 neighborhood differences in descriptive cooperative norms. That is, individuals who perceived

312 cheating to be common were more likely to steal, and stole more in the game. These individuals

313 tended to reside in Neighborhood B. Thus, the perception that others in the community are

314 cheating may have induced further antisocial behavior in the game. While this observation is

315 purely correlational, it is in accordance with the experimental results of Falk \& Fischbacher

316 (2002), who demonstrated a positive effect of observed theft on a participant's subsequent choice

317 to steal in the lab. It is also in agreement with those of Cialdini, Reno \& Kallgren (1990) and

318 Keizer, Lindenberg \& Steg (2008), who showed that observed norm violation can result in an 
increase in norm violation.

320 Correspondingly, participants in Neighborhood B indicated far less trust in their neighbors

321 than did those in A. This result fits with the far lower self-reported social capital in Neighborhood

322 B previously observed. Our measure of trust in the current study, trust neighbors, approximates

323 one of six items in the social capital index of Nettle, Colléony \& Cockerill (2011), which was

324 highly positively correlated with the overall index $(0.77$, p-value $<0.05)$.

325 As expected, and in concordance with social disorganization theory, trust neighbors was a

326 positive predictor of punitiveness. Kocher, Martinsson \& Visser similarly found that trust in

327 members of a participant pool was positively correlated with punitiveness in a public goods game

328 (2012). Although they interpreted this outcome as stemming from greater disappointment in free-

329 riding behavior, they suggest it merits further investigation of the role of social capital in norm

330 enforcement.

331 One possible interpretation of the unpredicted interaction we observed between trust

332 neighbors and value $£ 2$ lies in consideration of the multiple ways in which the cost of punishing

333 can vary for the punisher. We showed that participants were more punitive when value $£ 2$ was

334 smaller. Punitiveness is also less costly when there are fewer defectors and/or more punishers

335 (Boyd et al. 2003; Gürerk, Irlenbusch \& Rockenbach 2006; Boyd, Gintis \& Bowles 2010). Trust

336 neighbors may be informative as to whether Player 3 thinks there are many punishers and

337 defectors in her neighborhood and thus construed as a measurement of the cost of intervening in

338 antisocial behavior. From this perspective, our results are consistent with the idea that people are

339 more punitive when punishment is cheap - with respect to both material resources and the

340 behavior of others. This also highlights a limitation of this study, which is that Player 3 was able

341 to punish anonymously and therefore 'cheaply' with respect to possible retribution. In the real

342 world, third-party punishment may be associated with risk of retribution or other costs that are

343 not captured by the $£ 2$ Player 3 paid to exact punishment. Decreased resiliency to retribution 
344 could also vary by neighborhood, perhaps partly as a result of differing material resources.

345 We are unable to determine whether participants in Neighborhood B stole more money

346 than those in Neighborhood A because they thought punishment was less likely. This is because a

347 participant's motivation to steal a particular amount of money can be ascribed to inequity

348 aversion as well as the expected probability of punishment. However, our data from Player 2

349 address expectation of punishment. While we did not observe a robust neighborhood difference in

350 expect $3 P P$, we did observe a strong negative relationship between perceived cheating and expect

$3513 P P$. That is, a participant who thought many of her neighbors cheat on public goods was less

352 likely to expect a neighbor to pay $£ 2$ to fine Player 1 if Player 1 took half her money.

353 This result supports the idea that descriptive cooperative norms are indeed informative as

354 to expectation of punishment (Traxler \& Winter 2012). It also suggests that expectation of

355 punishment is one of the mechanisms by which signs of norm violation can lead to further

356 violation (Traxler \& Winter 2012; Kelling \& Wilson 1982). However, the causality of the

357 observed relationship between perceived cheating and expect 3PP remains unknown. Surveys of

358 the kind in Study 1 can only establish correlation; examining the causal significance of one

359 variable for another requires experimental manipulation of the first variable. With this in mind,

360 we undertook Study 2, in which we used selective feedback from Study 1 to experimentally alter

361 perceptions of perceived cheating in the two neighborhoods.

363 Study 2.

364 Feedback on or manipulation of descriptive norms has been used to alter people's behavior - in

365 diverse domains from littering (Cialdini, Reno \& Kallgren 1990) to energy use (Nolan et al.

366 2008). In Study 2, we used a novel method for manipulation of descriptive norms to investigate

367 the causality of the relationship between perceived cheating and expect $3 P P$. In each

368 neighborhood, we provided novice Player 2s with information on what their neighbors thought 
369 about the descriptive cooperative norms of the neighborhood ('Norms treatment'). We

370 manipulated this information so as to present Study 2 participants from Neighborhood A with a

371 less positive picture of descriptive norms than was really the case, and participants from

372 Neighborhood B with a more positive picture. We predicted that participants in Neighborhood A

373 who received the Norms treatment would be less likely to expect Player 3 to 3PP on their behalf,

374 compared to those participants in the same neighborhood who did not receive the treatment. We

375 predicted the opposite effect in Neighborhood B.

376

377 Study 2 Methods.

378 Sampling. We collected data for Study 2 from October to December 2012, while Study 1 was

379 ongoing (SI), following the same protocol as in Study 1.

380

381 Norms Questionnaire. We refer to the questionnaire used in Study 1 as 'Baseline treatment'. The

382 questionnaire for the Norms treatment differed as follows.

383 Civic norms manipulation: perceived cheating. The Norms questionnaire did not include

384 questions about injunctive and descriptive norms. We presented participants with information on

385 the responses of a subset of Study 1 participants in their neighborhood to the questions about

386 descriptive civic norms (SI).

387 The following backstory was used: As a part of the Tyneside Neighbourhoods Project, we

388 had asked 10 people in their neighborhood how common they think avoiding a public transport

389 fare, cheating the benefits system, and cheating on taxes, are in that neighborhood. We averaged

390 these answers to get an idea of how common people think certain behaviors are. We wanted to

391 know what other people in the neighborhood thought of these answers, and thus were asking

392 them (SI).

393 We presented one scale for each of the behaviors. The information in each scale was 
394 manipulated: in Neighborhood A, we took the mean of the 10 responses that gave the least

395 favorable impression of cheating (i.e., high perceived cheating), and in Neighborhood B, we took

396 the mean of the 10 responses that gave the most favorable impression of cheating (i.e., low

397 perceived cheating). The information presented for Neighborhood A was: 5.7 for avoid a fare on

398 public transport, 5.5 for cheat the benefits system, and 6.7 for cheat taxes (where $1=$ 'No one

399 would' and 10 = 'Everyone would'). In Neighborhood B the information presented was: 2.2 for

400 avoid a fare, 2.3 for cheat benefits, and 1.7 for cheat taxes. Beneath each scale, Study 2

401 participants were asked to circle 'Fewer people would do this,' 'This is about right,' or 'More

402 people would do this' (SI).

403 Contamination. To assess whether participants knew Study 1 participants, we included a

404 contamination question: 'Do you know of other people in your neighborhood who got a

405 questionnaire and plan to post it or already have posted it?' ('Yes', 'Not sure', or 'No').

406

407 3PP game. For Study 2, we measured the following behavior: expect 3PP (yes or no; representing 408 whether Player 2 expected Player 3 to punish Player 1 if Player 1 took $£ 5$ from her).

410 Statistical analyses. We used binary logistic regression to assess the effect of the Norms

411 treatment on expect $3 P P$ within each neighborhood.

412

413 Study 2 Results.

414 Participants. For Study 2, we sampled 41 participants from Neighborhood A (21 male) and 39

415 participants from B (16 male) (Table S2; SI).

416

417 Reaction to normative information. Participants in Neighborhood B were far more likely than

418 those in A to indicate 'This is about right' when presented with the manipulated norms scales for 
cheat benefits and avoid fare (OR 3.63, 95\% CI 1.23-10.70 and OR 3.74, 95\% CI 1.34-10.49,

420 respectively). In Neighborhood B, $38.46 \%, 43.59 \%$, and $46.15 \%$ of participants indicated 'This is

421 about right' for cheat benefits, avoid fare, and cheat taxes, respectively. In contrast, the majority

422 of participants in Neighborhood A indicated 'Fewer people would do this' when presented with

423 the manipulated scales for cheat benefits and avoid fare (78.05\% of participants for each

424 behavior). Only $51.28 \%$ of participants in Neighborhood A indicated 'Fewer people would do

425 this' for cheat taxes.

426

427 Expectation of 3PP: Norms treatment. Participants in Neighborhood B who received the Norms

428 treatment - i.e., who received information that their neighbors perceive cheating to be uncommon

429 - were more likely to expect Player 3 to 3PP on their behalf, compared to those in B who

430 received the Baseline treatment. The proportion of participants who expected 3PP is $58.97 \%$ for

431 the Norms treatment, compared to $30.23 \%$ for Baseline (OR 3.32, 95\% CI 1.33-8.25; Fig. 6).

432 Exclusion of participants for whom contamination was 'Not sure' (five) or 'Yes' (two) does not

433 qualitatively change the results. (One participant circled both.)

$434 \quad$ We did not observe a robust effect of the Norms treatment on expect $3 P P$ in

435 Neighborhood A. Contrary to our prediction, the proportion of participants in A who expected

$4363 \mathrm{PP}$ is $41.46 \%$ for Norms treatment, compared to $36.36 \%$ for Baseline treatment (OR 1.24, $95 \%$

437 CI 0.52-3.00; Fig. 6).

438 However, the Norms treatment generated an unanticipated response in Neighborhood A.

439 Some participants attempted to redirect their money by asking us to: donate it to charity (three

440 participants), keep it for research/university funds (two participants), or not pay them (one

441 participant). The rate of 'opting out of payment' is $14.63 \%$ for Norms treatment participants in

442 Neighborhood A, compared to $1.15 \%$ of Baseline participants in A (OR 11.25, 95\% CI 2.18-

443 57.97). This spontaneous change in game play was not observed in Neighborhood B. 


\section{Study 2 Summary and Discussion.}

446 In Study 2, participants in Neighborhood B received information that their neighbors think there

447 is little cheating on public goods in their neighborhood, relative to what we actually observed in

448 Study 1. They were far more likely to expect a neighbor to punish antisocial behavior compared

449 to those in Neighborhood B who did not receive the manipulation. Whether disorder can play a

450 causal role in an increase in crime rates (Kelling \& Wilson 1982) has been debated (Sampson,

451 Morenoff \& Gannon-Rowley 2002; Markowitz et al. 2001). Our results provide empirical

452 evidence of a mechanism by which norm violation can lead to the further violation of a different

453 norm - through change in the expectation of punishment.

454 There are at least three plausible routes by which this effect is achieved. One possibility is

455 that people expect cooperators to be more likely than non-cooperators to punish. The second is

456 that people perceive other's behavior to reflect other's expectation of punishment. That is, people

457 think that others are not behaving antisocially because of their expectations of punishment for

458 behaving antisocially. The third possibility, closely related to the second, is that if antisocial

459 behavior is very common, people may intuit that it persists because antisocial behavior is going

460 unpunished and thus have a decreased expectation of punishment.

461 We did not observe a reliable negative effect of the norms manipulation on expectation of

$4623 \mathrm{PP}$ in Neighborhood A. It is not clear why we observed the expected result in Neighborhood B

463 and not Neighborhood A. In Study 1, we found greater variation in trust and norms in

464 Neighborhood B than in Neighborhood A (Table 1). One interpretation of this is that the

465 environment is more heterogeneous and unpredictable in Neighborhood B. If so, perhaps

466 residents of Neighborhood B are less certain than residents of Neighborhood A of the behavior of

467 their neighbors and therefore were more accepting of the manipulation. Indeed, far more

468 Neighborhood B participants circled 'This is about right' when presented with the manipulated 
descriptive norms. Another possibility is that participants in Neighborhood B were more

470 accepting of the information provided by an authority figure (university personnel/scientist).

471

\section{General Discussion.}

473 The aim of this paper was to consider how the local social environment affects individual

474 decisions to engage in and sanction antisocial behavior, and how an individual's antisocial

475 behavior can in turn affect the local social environment, by conveying information about

476 descriptive norms. In Study 1, we observed that subjects in Neighborhood B took more money

477 from their neighbors and were less punitive in an economic game of crime and punishment. The

478 perception that others are cheating on public goods varied dramatically by neighborhood, was

479 fundamental to the neighborhood difference in theft in the game, and was negatively associated

480 with the expectation of third party punishment for antisocial behavior. Subjects in Neighborhood

481 B were also less punitive of antisocial behavior, and punitiveness was negatively associated with

482 trust in one's neighbors.

483 In Study 2, we showed that providing participants in Neighborhood B with information

484 that cheating is perceived as uncommon within their neighborhood led to a sharp increase in the

485 expectation of third-party punishment for theft. An increase in the perceived likelihood of

486 punishment would presumably lead to greater cooperation, given the close relationship between

487 these two variables. Thus, these results provide novel empirical support for a mechanism by

488 which cues of norm violation can lead to further norm violation (Cialdini, Reno \& Kallgren 1990;

489 Keizer, Lindenberg \& Steg 2008): altered expectation of punishment (Kelling \& Wilson 1982;

490 Traxler \& Winter 2012).

491 We consider these results within a framework where culture is dynamic, subject to

492 evolutionary processes that can lead to more or less cooperative outcomes (Boyd \& Richerson

493 1985). Unlike in recent cross-cultural studies of cooperation and punishment (Henrich et al. 
494 2006; Herrmann, Thöni \& Gächter 2008), our two study populations share many cultural

495 components, including the institutions that formally sanction their civic violations (although how

496 those institutions are experienced may vary) and injunctive cooperative norms. The apparently

497 large discrepancy between desired and achieved cooperative outcomes in Neighborhood B, as

498 assessed with injunctive and descriptive cooperative norms, adds a new perspective on the

499 cultural evolution of variable cooperative outcomes.

$500 \quad$ Our results provide evidence for three potential routes by which perceived cooperative

501 norm violation can lead to further violation of cooperative norms ${ }^{1}$. All of these processes have

502 been postulated or investigated by others; however, to our knowledge, they have not been

503 considered simultaneously as processes that may, in concert, lead to substantial cultural change.

504 These positive feedback processes are: 1) To avoid being 'suckered', conditional cooperators are

505 motivated to defect if they perceive that defection is common (Gächter \& Fehr 2001; Falk \&

506 Fischbacher 2002; Bichierri and Xiao 2009; Raihani \& Hart 2010; Fischbacher, Irwin \& Simpson

507 2013). 2) Perceived cheating leads to lower trust. Low trust results in reduced informal

508 punishment of norm violation (Kocher, Martinsson \& Visser 2012). In this vein, Traxler and

509 Winter (2012) observe a direct effect of the perceived frequency of norm violations on expressed

510 willingness to sanction violations. Similarly, extensions of social disorganization theory include

511 feedback processes between crime/disorder and social cohesion/control, via fear or residential

512 instability (Sampson \& Raudenbush 1999; Markowitz et al. 2001; Steenbeck \& Hipp 2011). 3)

513 When the perceived frequency of cooperative norm violation is high, expectation of punishment

514 for violation is lower (Sah 1991; Traxler and Winter 2012).

515 We hypothesize that these three positive feedback mechanisms, wherein perceived

516 cooperative norm violation leads to further cooperative norm violation, could act simultaneously

1 Our studies focused on cooperative norm violation ("perceived cheating”) and a very specific type of antisocial behavior (theft). However, based on work on cross-norm effects referenced in the introduction, we think that we can draw inferences here not just about theft but cooperative norm violation in general. 
517 to result in a rapid downward spiral, leading to low levels of cooperation. As Cialdini, Reno \&

518 Kallgren (1990) note, descriptive norms are informative as to adaptive behavior. In a community

519 with low levels of cooperation and minimal punishment of cooperative norm violation, non-

520 cooperative strategies may outperform others (Wilson \& Csikszentmihalyi 2007). Other

521 processes - prestige-biased (Henrich \& Gil-White 2001) or conformist (Henrich \& Boyd 1998)

522 transmission and self-selection of people with preferences for an antisocial community - could

523 further reinforce uncooperative or overtly antisocial strategies. While cooperative norms are

524 considered a component of social capital (Knack \& Keefer 1997; Bowles \& Gintis 2002), our

525 results demonstrate the need for explicit integration of cultural transmission and norm

526 psychology - i.e., psychological adaptations for determining and adopting local norms and

527 punishing violators (Chudek \& Henrich 2011) - with social disorganization theory. Scholars of

528 criminology will note some similarities between the social learning theory of deviance (Akers

529 2009) and theories of cultural transmission. However, we extend this bridge between the social

530 environment and individual behavior by emphasizing the feedback from the individual to the

531 social group. That is, we have outlined three routes by which an individual's defection can lead

532 other individuals to adopt similar behavioral strategies, thus altering the local cultural ecology

533 (Camerer \& Fehr 2006).

534 Missing from this hypothesized downward spiral is an initial perturbation that could result

535 in an increase in cooperative norm violation (or perceived violation) in the neighborhood.

536 Poverty and economic uncertainty are also striking differences between Neighborhoods A and B.

537 Without middle class buffers of savings and credit, institutional safety nets, or strong reciprocal

538 networks, crises such as illness create the potential for dire outcomes, thus altering the costs and

539 benefits of defecting. For people already living at the margin, material crises might result in a

540 higher probability of defection. Especially for crises that hit broad swaths of a community

541 simultaneously, such as the widespread job loss in Neighborhood B resulting from the collapse of 
542 the shipbuilding and coal mining industries, one can imagine an increase in the frequency of

543 defection that alters the descriptive cooperative norms enough to start a downward spiral in

544 defection.

545 Importantly, although we hypothesize that poverty and economic uncertainty were linked

546 to an initial perturbation of cooperative norm violation in the current study, the positive feedback

547 of norm violation could continue in the absence of poverty. There has been debate as to whether

548 there are direct, as well as indirect, effects of poverty and/or income inequality on crime

549 (Patterson 1991; Bursik \& Grasmick 1993). The story we have sketched is compatible with both

550 possibilities, as an historical direct effect of poverty on norm violation may lead to cultural

551 dynamics that persist beyond the duration of the poverty itself. (For a similar example of such

552 cultural inertia, see Sah (1991), who argues that a transient change in the economics of crime can

553 lead to persistently high crime rates, due to a postulated relationship between higher crime rates

554 and decreased expectation of punishment.)

555 However, we can only speculate as to whether these dynamics are at play in Neighborhood B

556 (outside of the 3PP game) and to what extent they can explain the observed high rates of crime

557 and antisocial behavior.

558 This paper also makes contributions to empirical gaps in two fields. In Study 1, we

559 demonstrated that the covariation of cooperation and punishment of non-cooperation, which has

560 been observed cross-culturally with economic games (Henrich et al. 2006), can extend to the

561 local level. Participants in Neighborhood A stole less money and were more punitive in the game

562 than those in B. Also in Study 1, we demonstrated an association - albeit small - between third-

563 party punishment of antisocial behavior and trust in one's neighbors, as well as a neighborhood-

564 level association between antisocial behavior in the game and decreased third-party punishment

565 of antisocial behavior. These results provides additional, novel empirical support for the

566 relationship between 1) low social control and low social capital, and 2) low social control and 
567 high rates of antisocial behavior. Data on actual social control (rather than the potential that

568 residents will engage in social control, as measured by survey data) are difficult to come by,

569 limiting the strength of the inference that low social capital and high rates of antisocial behavior

570 are correlated due to lack of social control (Bursik \& Grasmick 1993; Steenbeck \& Hipp 2011).

571 We acknowledge that there are a number of limitations to our studies. We could not

572 control the order at which participants looked at or filled out packet components. It is possible

573 that participants 'justified' their behavior in the game with their questionnaire answers. However,

574 we might then expect a robust positive effect of value $£ 1$ on theft. Presenting Player 1 s with the

575 threat of punishment for theft could have decreased intrinsic motivation to behave cooperatively

576 (Frey \& Jegen 2001), although it is unclear how this would produce a spurious correlation

577 between perceived cheating and theft in the game. We cannot account for the neighborhood

578 residents who chose not to respond, although in both neighborhoods we likely reached a segment

579 of the community biased towards prosocial preferences (registered voters and research

580 participants). Additionally, although participants were anonymous to each other in the game, they

581 were not anonymous to us. The neighborhood differences in game behavior we observed could be

582 partly attributed to participants in Neighborhood A, but not Neighborhood B, regarding a

583 university professor as someone in their social milieu and thus being concerned about

584 reputational repercussions.

$585 \quad$ Finally, we have two related suggestions for future study that may increase our

586 understanding of why some communities appear to be stuck at uncooperative equilibria, despite

587 concerted efforts by city planners to chart a different course (Robinson 2005), or even substantial

588 temporal changes in the demographic makeup (Shaw \& McKay 1942). The first is further

589 investigation of the potential for simultaneous multiple paths of positive feedback on cooperative

590 norm violation, including not just conditional cooperation but also punitiveness and expectation

591 of punishment. The second is consideration of how psychological adaptations for recognizing and 
592 adopting local norms, as well as biased in- and out-migration (Chudek \& Henrich 2011), can

593 reinforce an antisocial culture.

594

595 
596 Acknowledgments.

597 We thank residents of Newcastle Upon Tyne for participating in these studies, and we thank M.N.

598 Grote, R. McElreath, and K. Rauch for helpful discussion and comments. 
599

600 Akaike H. 1974. A new look at the statistical model identification. IEEE Transactions on

601

602

603

604 Bowles S, Gintis H. 2002. Social capital and community governance. The Economic Journal 605

606

607

608

609

610

611

612

613

614 Bursik RJ, Grasmick HG. 1993. Economic deprivation and neighborhood crime rates, 1960-

615

616 Camerer CF, Fehr E. 2004. Measuring social norms and preferences using experimental games:

617 a guide for social scientists. In: Henrich J, Boyd R, Bowles S, Camerer C, Fehr E, Gintis H, eds.

618 Foundations of Human Sociality: Economic Experiments and Ethnographic Evidence from

619 Fifteen Small-Scale Societies. New York: Oxford University Press, 55-95.

620 Camerer CF, Fehr E. 2006. When does "Economic Man" dominate social behavior? Science

621

622 Chudek M, Henrich Joseph. 2011. Culture-gene coevolution, norm-psychology and the 

10.1016/j.tics.2011.03.003.

625 Cialdini RB, Reno RR, Kallgren CA. 1990. A focus theory of normative conduct: recycling 626 the concept of norms to reduce littering in public places. Journal of Personality and Social Psychology 58:1015-1026. doi: 10.1037//0022-3514.58.6.1015.

628 Ecklund A. 2012. beeswarm: The bee swarm plot, an alternative to stripchart. Available at 629 http://cran.r-project.org/package=beeswarm.

630 Falk A, Fischbacher U. 2002. "Crime" in the lab - detecting social interaction. European Economic Review 46:859-869. doi: 10.1016/S0014-2921(01)00220-3.

632 Fehr E, Fischbacher U. 2004. Third-party punishment and social norms. Evolution and Human 633 Behavior 25:63-87.

634 Fehr E, Gächter S. 2000. Cooperation and punishment in Public Goods experiments. 635 American Economic Review 90:980-994.

636 Fischbacher U, Gächter S, Fehr E. 2001. Are people conditionally cooperative? Evidence from a 637 public goods experiment. Economics Letters 71:397-404.

638 Frey BS, Jegen R. 2001. Motivation crowding theory. Journal of Economic Surveys 15:589639 611. doi: 10.1111/1467-6419.00150.

640 Glaeser EL, Sacerdote B, Scheinkman JA. 1996. Crime and social interactions. Quarterly $641 \quad$ Journal of Economics 11:507-548.

642 Gürerk O, Irlenbusch B, Rockenbach B. 2006. The competitive advantage of sanctioning 643 institutions. Science 312:108-11. doi: 10.1126/science.1123633.

644 Henrich J, Boyd R. 1998. The evolution of conformist transmission and the emergence of 645 between-group differences. Evolution and Human Behavior 19:215-241.

646 Henrich J, McElreath R, Barr A, Ensminger J, Barrett C, Bolyanatz A, Cardenas JC, Gurven 

punishment across human societies. Science 312:1767-1770.

649 Henrich J, Gil-White FJ. 2001. The evolution of prestige: freely conferred deference as a 650 mechanism for enhancing the benefits of cultural transmission. Evolution and Human 651 Behavior 22:165-196. doi: 10.1016/S1090-5138(00)00071-4.

652 Herrmann B, Thöni C, Gächter S. 2008. Antisocial punishment across societies. Science $653 \quad 319: 1362-7$. doi: $10.1126 /$ science. 1153808.

654 Irwin K, Simpson B. 2013. Do descriptive norms solve social dilemmas? Conformity and 655 contributions in collective action groups. Social Forces 91:1057-1084. 656 doi: $10.1093 /$ sf $/$ sos 196.

657 Keizer K, Lindenberg S, Steg L. 2008. The spreading of disorder. Science 322:1681-5.

658 Keizer K, Lindenberg S, Steg L. 2013. The Importance of Demonstratively Restoring Order. PLoS ONE 8(6): e65137. doi:10.1371/journal.pone.0065137.

660 Kelling BGL, Wilson JQ. 1982. Broken Windows. The Atlantic Monthly March:29-38.

661 Knack S, Keefer P. 1997. Does social capital have an economic payoff? A cross-country 662 investigation. Quarterly Journal of Economics 112:1251-1288.

663 Kocher M, Martinsson P, Visser M. 2012. Social background, cooperative behavior, and norm 664 enforcement. Journal of Economic Behavior \& Organization 81:341-354. doi: 665 10.1016/j.jebo.2011.10.020.

666 Lamba S, Mace R. 2011. Demography and ecology drive variation in cooperation across human 667 populations. Proceedings of the National Academy of Sciences of the United States of 668 America 108:14426-30. doi: 10.1073/pnas.1105186108.

669 Lochner L. 2007. Individual perceptions of the criminal justice system. American Economic $670 \quad$ Review 91:444-460. 
671 Markowitz FE, Bellair PE, Liska AE, Liu J. 2001. Extending social disorganization theory:

672 modeling the relationships between cohesion, disorder, and fear. Criminology 39:293-

$673 \quad 320$.

674 Mcelreath R. 2012. rethinking: Statistical Rethinking book package. Available at

675 https://github.com/rmcelreath?tab=repositories

676 Nettle D, Colléony A, Cockerill M. 2011. Variation in cooperative behaviour within a single

677 city. PloS one 6:e26922. doi: 10.1371/journal.pone.0026922.

678 Nettle D, Pepper GV, Jobling R, Schroeder KB. 2014. Being there: a brief visit to a

679 neighbourhood induces the social attitudes of that neighbourhood. PeerJ 2:e236

$680 \quad$ http://dx.doi.org/10.7717/peerj.236.

681 Nolan JM, Schultz PW, Cialdini RB, Goldstein NJ, Griskevicius V. 2008. Normative social

682 influence is underdetected. Personality \& Social Psychology Bulletin 34:913-23.

683 doi: $10.1177 / 0146167208316691$.

684 Patterson EB. 1991. Poverty, income inequality, and community crime rates. Criminology

685 29:755-776. doi: 10.1111/j.1745-9125.1991.tb01087.x.

686 Raihani NJ, Hart T. 2010. Free-riders promote free-riding in a real-world setting. Oikos

687 119:1391-1393. doi: 10.1111/j.1600-0706.2010.18279.x.

688 Robinson F. 2005. Regenerating the West End of Newcastle: what went wrong? Northern

$689 \quad$ Economic Review 36:15-41.

690 Sah RK. 1991. Social osmosis and patterns of crime. Journal of Political Economy 99:1272-

$691 \quad 1295$.

692 Sampson R. J., Raudenbush S. W., Earls F. 1997. Neighborhoods and violent crime: A

693 multilevel study of collective efficacy. Science 277:918-924.

694 doi:10.1126/science.277.5328.918. 
695 Sampson Robert J, Raudenbush Stephen W. 1999. Systematic social observation of public

696 spaces: a new look at disorder in urban neighborhoods. American Journal of

697 Sociology 105:603-651.

698 Sampson Robert J., Groves WB. 1989. Community structure and crime: testing social-

699 disorganization theory. American Journal of Sociology 94:774-802.

$700 \quad$ doi:10.1086/229068.

701 Sampson Robert J., Morenoff JD, Gannon-Rowley T. 2002. Assessing "neighborhood

702 effects": social processes and new directions in research. Annual Review of

703

Sociology 28:443-478. doi: 10.1146/annurev.soc.28.110601.141114.

704 Shaw C, McKay H. 1942. Juvenile Delinquency and Urban Areas. Chicago, IL: University of

$705 \quad$ Chicago Press.

706 Steenbeck W, Hipp JR. 2011. A longitudinal test of social disorganization theory: feedback

707 effects among cohesion, social control, and disorder. Criminology 49:833-871. doi:

$708 \quad$ 10.1111/j.1745-9125.2011.00241.x.

709 R Core Team. 2012. R: A language and environment for statistical computing. Austria: R

$710 \quad$ Foundation for Statistical Computing. Available at http://www.R-project.org

711 Traxler C, Winter J. 2012. Survey evidence on conditional norm enforcement. European

712 Journal of Political Economy 28:390-398. doi: 10.1016/j.ejpoleco.2012.03.001.

713 Venables WN, Ripley BD. 2002. Modern Applied Statistics with S. Fourth edition. New York:

$714 \quad$ Springer.

715 Wickham H. 2009. ggplot2: elegant graphics for data analysis. New York: Springer.

716 Wilson DS, Csikszentmihalyi M. 2007. Health and the ecology of altruism. In: Post SG, ed.

717 Altruism and Health: Perspectives from Empirical Research. Oxford: Oxford

$718 \quad$ University Press, 314-331.

719 Wilson DS, O’Brien DT, Sesma A. 2009. Human prosociality from an evolutionary 
722 Yamagishi T. 1986. The provision of a sanctioning system as a public good. Journal of

723 Personality and Social Psychology 51: 110-116.

725

726

727

728

729

730

731

732 


\section{Figure 1}

Player 3 behavior, punitiveness, by neighborhood.

Each diamond represents one observation.

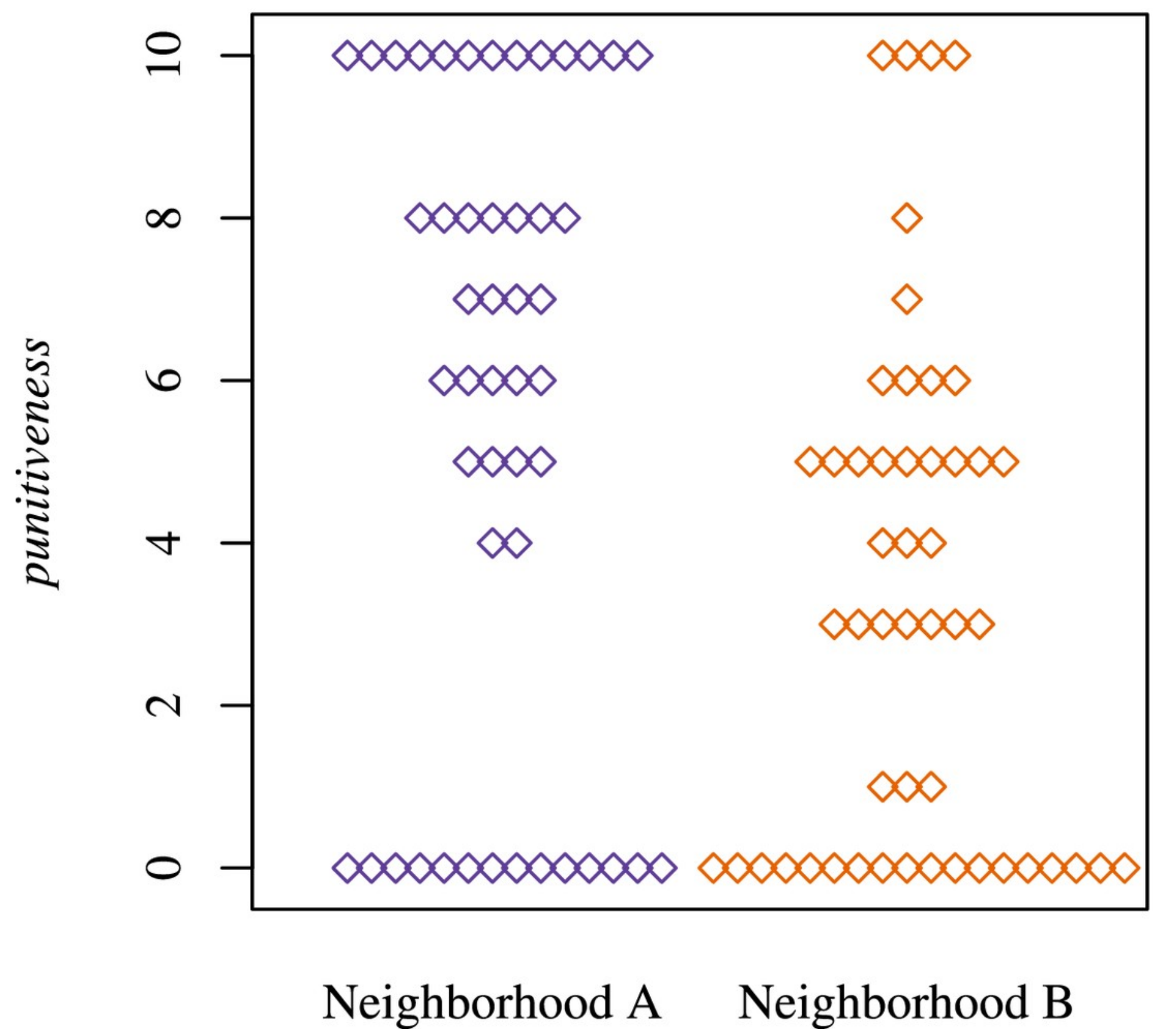




\section{Figure 2}

Punitiveness modeled as an interaction between trust neighbors and value $£ 2$.

Blue is 'high trust' (8; median trust neighbors score for Neighborhood A). Orange is 'low trust' (5; median trust neighbors score for Neighborhood B). Dotted lines are $95 \%$ confidence intervals.

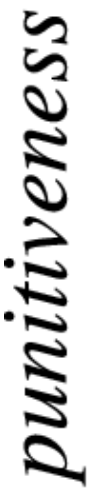

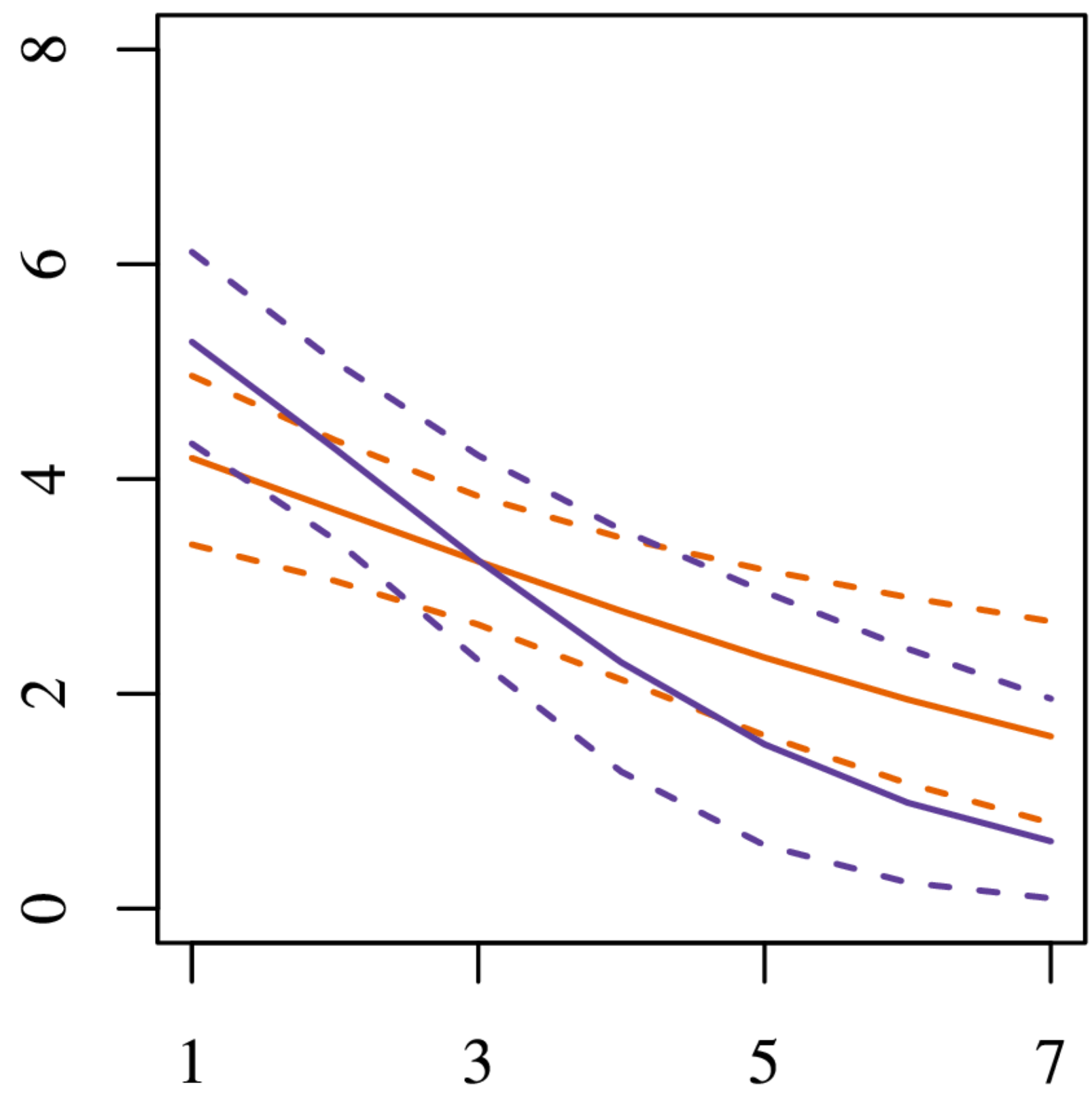

value $£ 2$ 


\section{Figure 3}

Neighborhood means and standard errors for condoned cheating and perceived cheating.

For condoned cheating, $1=$ Never OK, 10 = Always OK, and for perceived cheating, $1=$ No one would, 10 = Everyone would.

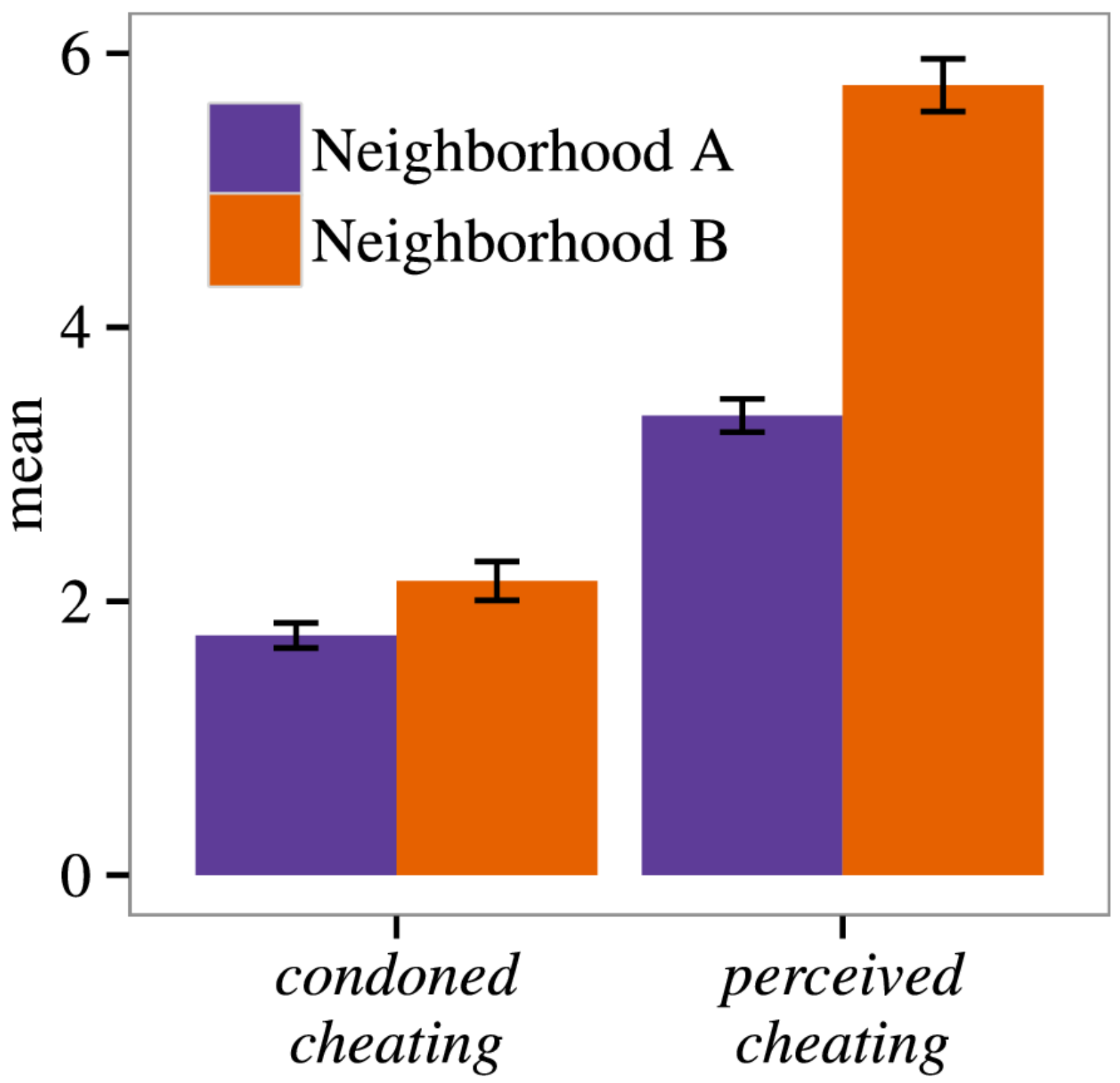




\section{Figure 4}

Theft for Player 1 modeled as dependent on perceived cheating.

Dotted lines are $95 \%$ confidence intervals. Bubbles represent the actual data from

Neighborhood A (blue) and Neighborhood B (orange). Size of the bubble corresponds to the number of observations.

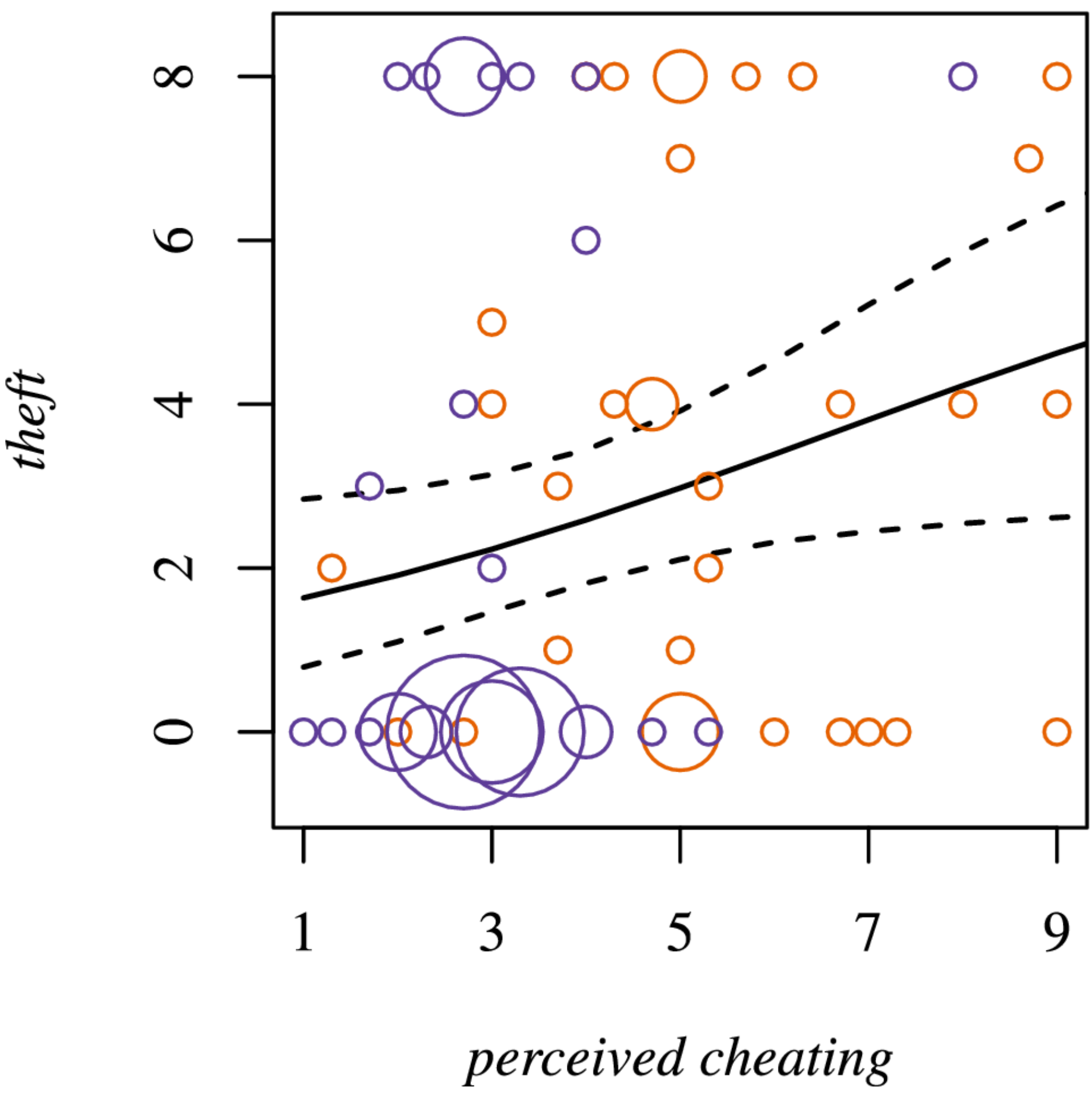




\section{Figure 5}

Probability of expect $3 P P$ dependent on perceived cheating.

Dotted lines are $95 \%$ confidence intervals.

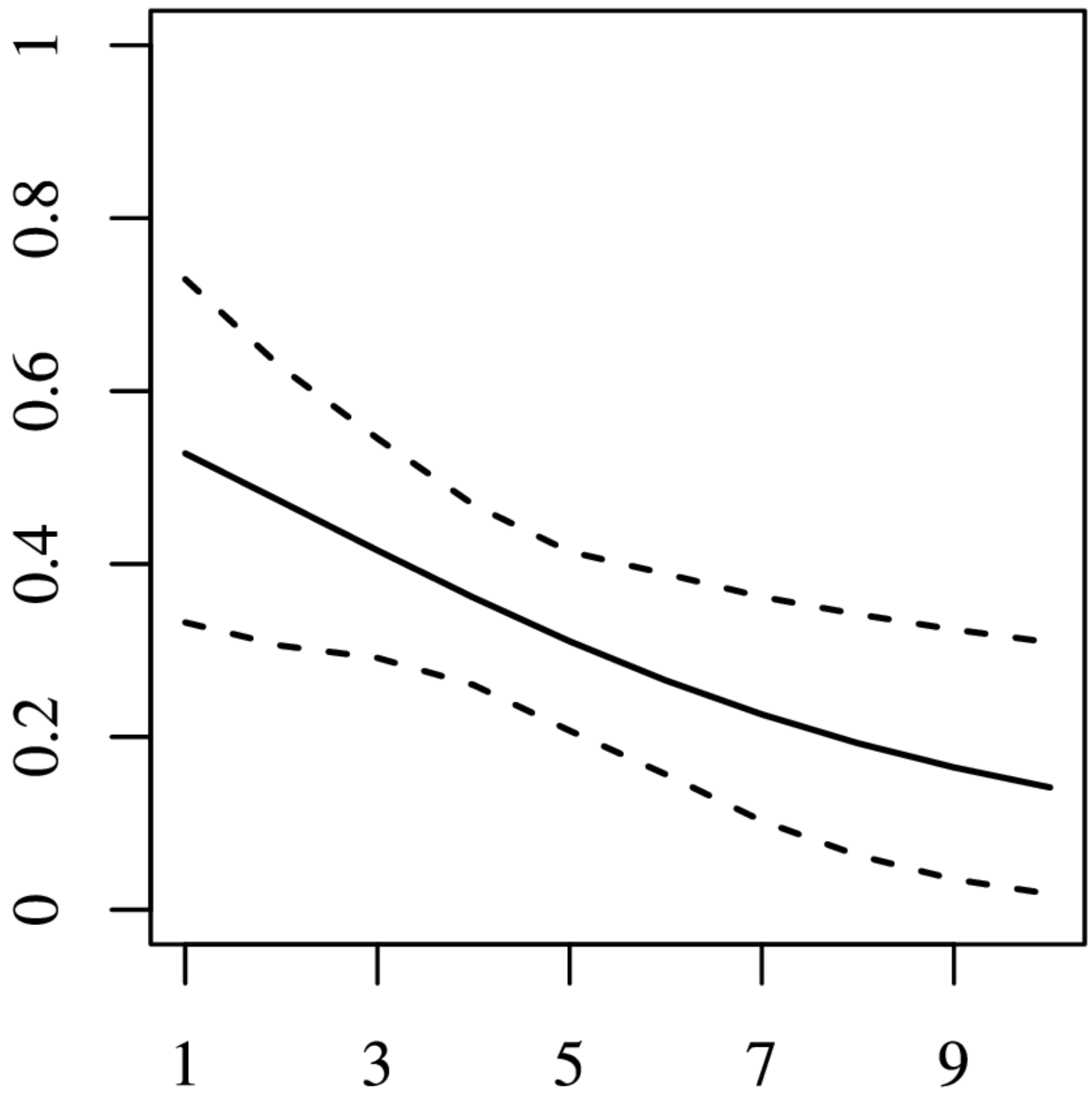

perceived cheating 


\section{Figure 6}

Proportion of Player 2s, by treatment and neighborhoood, who indicated that they expect Player 3 to 3PP on their behalf.

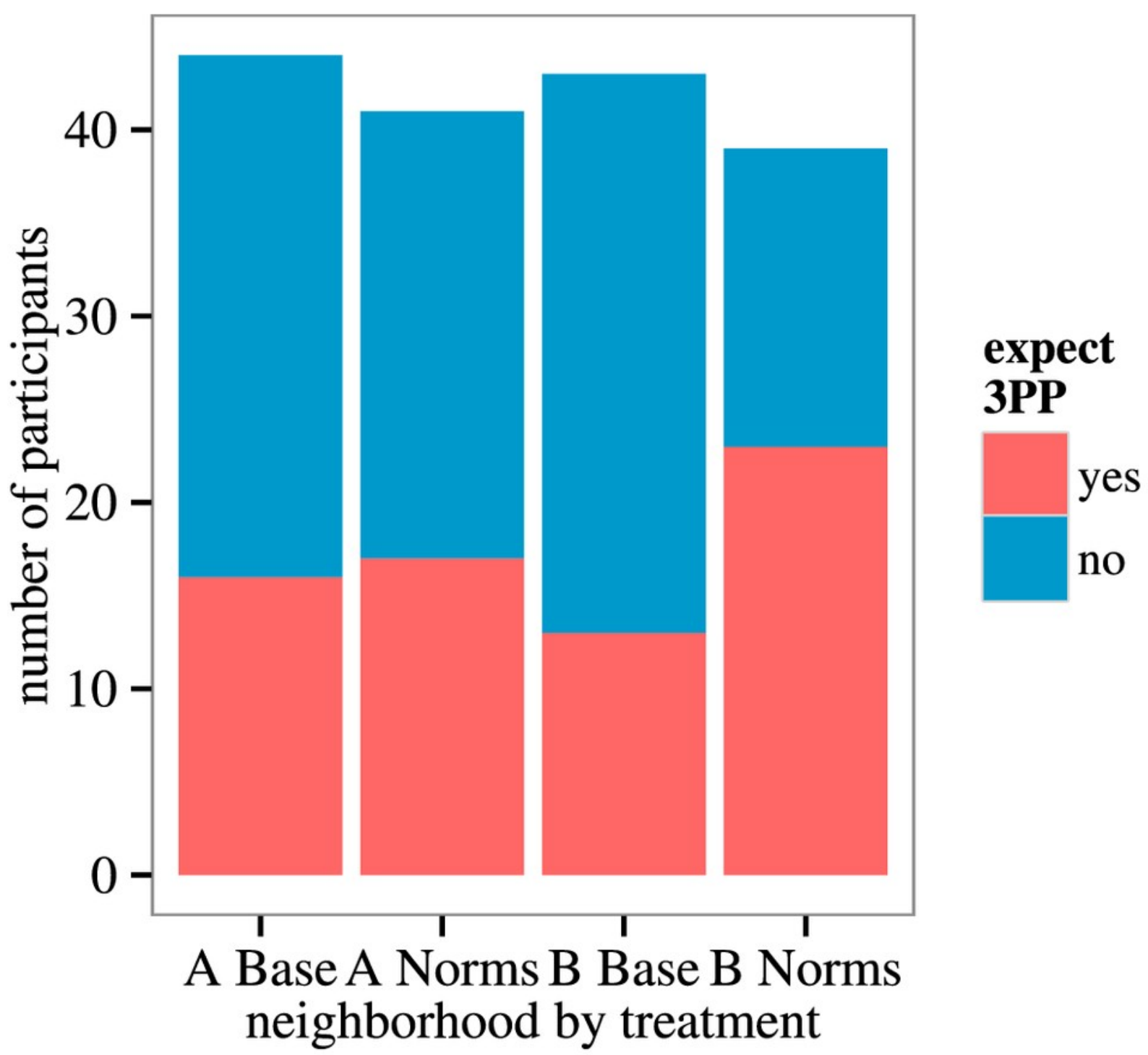




\section{Table 1 (on next page)}

Key variables from Study 1 by neighborhood.

Columns one and two contain medians for Neighborhood A and Neighborhood B, respectively (median absolute deviation in parentheses). Column three contains the odds that a participant from Neighborhood B indicated a higher value (95\% confidence interval in parentheses). Condoned cheating and perceived cheating are the mean of the three injunctive and descriptive norms, respectively. 


\begin{tabular}{|c|c|c|c|}
\hline Variable & $\stackrel{ }{\stackrel{ }{ }}$ Median A (scale 1 to 10) & Median B (scale 1 to 10) & Odds B higher \\
\hline trust neighbors & $8(1)$ & $5(2)$ & $0.053(0.031,0.093)$ \\
\hline value $£ 1$ & $\stackrel{\text { of }}{\supset} 1(0)$ & $1(0)$ & $1.89(0.77,4.61)$ \\
\hline value $£ 2$ & $\frac{\sqsubset}{\sigma} 1(0)$ & $2.5(1.5)$ & $5.53(2.51,12.18)$ \\
\hline value $£ 10$ & $\sum 3(1)$ & $5(3)$ & $3.37(1.04,10.9)$ \\
\hline injunctive avoid fare & ఠ $1(0)$ & $1(0)$ & $0.84(0.52,1.35)$ \\
\hline injunctive cheat benefits & $\cdot \bar{l} 1(0)$ & $1(0)$ & $1.43(0.87,2.34)$ \\
\hline injunctive cheat tax & $\frac{d}{>} 1(0)$ & $1(0)$ & $1.38(0.84,2.23)$ \\
\hline descriptive avoid fare & $3(1)$ & $6(2)$ & $11.02(6.58,18.46)$ \\
\hline descriptive cheat benefits & ए $3(1)$ & $6(2)$ & $13.95(8.16,23.85)$ \\
\hline descriptive cheat tax & $4(1)$ & $5(2)$ & $3.06(1.95,4.79)$ \\
\hline condoned cheating & (1) $1.33(0.33)$ & $1.50(0.50)$ & $1.25(0.80,1.95)$ \\
\hline perceived cheating & (1) $3.00(0.67)$ & $5.50(1.83)$ & $10.22(6.18,16.90)$ \\
\hline
\end{tabular}

